\title{
Temperature spectra in shear flow and thermal convection
}

\author{
Detlef Lohse * \\ October 29, 2018 \\ The James Franck Institute, The University of Chicago, \\ 5640 South Ellis Avenue, Chicago, IL 60637, USA
}

\begin{abstract}
We show that the $P_{u}(\omega) \propto \omega^{-7 / 3}$ shear velocity power spectrum gives rise to a $P_{\Theta}(\omega) \propto \omega^{-4 / 3}$ power spectrum for a passively advected scalar, as measured in experiment [K. Sreenivasan, Proc. R. Soc. London A 434, 165 (1991)]. Applying our argument to high Rayleigh number Rayleigh Benard flow, we can account for the measured scaling exponents equally well as the Bolgiano Obukhov theory (BO59). Yet, of the two explanations, only the shear approach might be able to explain why no classical scaling range is seen in between the shear (or BO59) range and the viscous subrange of the experimental temperature spectrum [I. Procaccia et al., Phys. Rev. A 44, 8091 (1991)].
\end{abstract}

PACS: 47.27.-i 


\section{Velocity shear spectra}

The classical scaling laws in fully developed turbulence are the Kolmogorov [1] and the Obukhov-Corrsin [2] scaling relations

$$
E_{u}(k) \sim \epsilon_{u}^{2 / 3} k^{-5 / 3}, \quad E_{\Theta}(k) \sim \epsilon_{\Theta} \epsilon_{u}^{-1 / 3} k^{-5 / 3}
$$

for the velocity spectrum $E_{u}(k)$ and the temperature spectrum $E_{\Theta}(k)$ of a passively advected temperature field. Here, $\epsilon_{u}$ and $\epsilon_{\Theta}$ are the energy and thermal dissipation rates, respectively. Eqs. (1) follow from dimensional analysis. They can also be derived from the assumption that the energy flux $T_{u}(k) \sim k u^{3}(k)$ of the conserved total energy $\int \boldsymbol{u}^{2}(\boldsymbol{x}) d \boldsymbol{x} / 2$ and the corresponding flux $T_{\Theta}(k) \sim k u(k) \Theta^{2}(k)$ of the conserved total temperature intensity $\int \Theta^{2}(\boldsymbol{x}) d \boldsymbol{x}$ do not depend on $k$, i.e., $T_{u}(k) \sim \epsilon_{u}$ and $T_{\Theta}(k) \sim \epsilon_{\Theta}$. Here we have assumed that both velocity and temperature fields are stirred only on the largest scales 1 and, of course, for these large scales we have neglected viscosity and diffusivity. $\boldsymbol{u}(\boldsymbol{k})$ and $\Theta(\boldsymbol{k})$ are the discrete Fourier transforms of the velocity and the temperature fields (having dimensions of length/time and temperature, respectively).

In anisotropic flow, a correction to (1) is expected. Lumley [4] was the first to suggest the spectrum

$$
E_{u}(k) \sim \epsilon_{u}{ }^{2 / 3} k^{-5 / 3}\left(1+\alpha_{u}\left(\frac{k}{k_{s}}\right)^{-2 / 3}\right)
$$

for an anisotropic flow, e.g., a shear flow. Here, $\alpha_{u}$ is an unknown dimensionless parameter and $k_{s}$ the crossover wave vector separating the shear dominated range (SDR) and the classical scaling range. Eq. (2) has also been obtained by dimensional analysis in terms of Clebsch variables [5, 6, 7].

In what follows we will be interested in situations where the second term in (2) dominates. This definitely is the case in cross spectra $E_{12}(k)$, which probe anisotropy. Indeed, $E_{12}(k) \propto k^{-7 / 3}$ was measured in boundary layer flow [8]. Also, Maloy and Goldburg's [9] scaling of the velocity structure function $D^{(2)}(r) \propto r^{4 / 3}$ in Taylor-Couette flow with an oscillatory inner cylinder might possibly be understood in terms of (2) with a large $\alpha_{u}$.

We also mention that $E_{u}(k) \propto k^{-7 / 3}$ scaling is expected in helical flow, i.e., flow for which the helicity $\int \boldsymbol{u} \cdot \boldsymbol{\nabla} \times \boldsymbol{u} d \boldsymbol{x}$ is non zero. The corresponding flux is then of order $T_{H e l}(k) \sim k^{2} u^{3}(k)$. Assuming that $T_{H e l}(k)=\epsilon_{H e l}$ does not depend on $k$ in a helicity dominated range, we obtain

$$
E_{u}(k) \sim \epsilon_{H e l}^{2 / 3} k^{-7 / 3} .
$$

The crossover $k_{s}$ to classical scaling (11) can be estimated as $k_{s}=\epsilon_{H e l} / \epsilon_{u}$.

\section{Temperature shear spectra}

How does the spectrum of a passive scalar (e.g., the temperature) look, if advected by a shear velocity field? From the conserved flux $T_{\Theta}(k) \sim k u(k) \Theta^{2}(k) \sim \epsilon_{\Theta}$ and the shear

\footnotetext{
${ }^{1} \mathrm{~A}$ different stirring force is discussed in [3].
} 
velocity scaling $u(k) \sim \epsilon_{u}{ }^{1 / 3} k_{s}^{1 / 3} k^{-2 / 3}$ we readily conclude $\Theta(k) \sim \epsilon_{\Theta}{ }^{1 / 2} \epsilon_{u}{ }^{-1 / 6} k_{s}^{-1 / 6} k^{-1 / 6}$ or

$$
E_{\Theta}(k) \sim \epsilon_{\Theta} \epsilon_{u}^{-1 / 3} k_{s}^{-1 / 3} k^{-4 / 3} .
$$

Considering also the high- $k$ range of the spectrum as in (2), we may write

$$
E_{\Theta}(k) \sim \epsilon_{\Theta} \epsilon_{u}^{-1 / 3} k^{-5 / 3}\left(1+\alpha_{\Theta}\left(\frac{k}{k_{s}}\right)^{-1 / 3}\right)^{-1} .
$$

The dimensionless constant $\alpha_{\Theta}$ will be estimated below.

Experimental support for our suggested scaling law (4) is given by Sreenivasan [10], who measured the power spectrum $P_{\Theta}(\omega)$ for temperature fluctuations in the wake of a heated cylinder for $R e_{\lambda}=200$. The power spectrum $P_{\Theta}(\omega)$ corresponds to $E_{\Theta}(k)$ via Taylor's hypothesis [1]]. Indeed, $P_{\Theta}(\omega) \propto \omega^{-4 / 3}$ for more than 1.5 decades, see fig. 1 . The long scaling range is surprising for the small $R e_{\lambda}$ as it was in [9]. Unfortunately, the velocity spectrum in this experiment [10] was not measured. For the cross spectrum one would expect $E_{12}(k) \propto k^{-7 / 3}$ as in the boundary layer [8]. We thus felt justified to assume $u(k) \propto k^{-2 / 3}$ here, as the flow behind the cylinder is strongly anisotropic.

Where should we expect the crossover between the SDR and the K41 range? We estimate the crossover frequency $\omega_{s}$ directly by the shear $s$. With the data given in [10], we find $\omega_{s}=s=U / L=885 \mathrm{sec}^{-1}$. Here, $U=1770 \mathrm{~cm} / \mathrm{s}$ is the flow velocity and $L=2 \mathrm{~cm}$ the diameter of the heated cylinder. Our result $\log _{10}\left(\omega_{s} s e c\right)=2.9$ is indeed very near the end of the $P_{\Theta}(\omega) \propto \omega^{-4 / 3}$ scaling range, cf. fig. 1. The K41 subrange in between the SDR and the viscous subrange (VSR) seems to be small, if it exists at all for this small $R e_{\lambda}$. Yet for larger $R e_{\lambda}$ it might show up in between SDR and VSR. Along this line of argument we can understand why the (effective) scaling exponent of $P_{\Theta}(\omega)$ approaches the classical value $-5 / 3$, when $R e_{\lambda}$ is increased, see fig. 6 of ref. [10.

To be more quantitative, we compare the $R e_{\lambda}$ dependence of $k_{s}$ and that of the viscous dissipation cutoff $k_{d}$. The latter is calculated to be $12 \pi k_{d}^{-1} \approx 18 L R e_{\lambda}{ }^{-3 / 2}$. For the former we estimate $k_{s}^{-1} \lesssim L$, as in this geometry shear is induced in the flow at some large length scale. So for increasing $R e_{\lambda}$, a classical scaling range can develop between the SDR $k<\alpha_{\Theta}^{3} k_{s}$ and the VSR $k>k_{d}$, which dominates the spectrum at large $k$. From the experimental observation that the K41 subrange is still missing for $R e_{\lambda}=200$ [10], we can estimate $\alpha_{\Theta}$ to be $\alpha_{\Theta}=\left(k_{d}\left(R e_{\lambda}=200\right) / k_{s}\right)^{1 / 3} \approx 5$. The classical scaling range will grow as $k_{d} /\left(\alpha_{\Theta}^{3} k_{s}\right)=\left(R e_{\lambda} / 200\right)^{3 / 2}$. For $R e_{\lambda}=1000$ it is already larger than one decade and more or less classical scaling exponents will be extracted from the temperature spectra, which accounts for the measurements summarized in fig. 6 of [10].

\section{Rayleigh-Benard flow}

It is tempting to apply our considerations also to Rayleigh-Benard (RB) flow, which has recently been studied in detail [13-19]. RB flow is governed by a large scale "wind"

\footnotetext{
${ }^{2}$ Strictly speaking, instead of the factor in brackets, one should write $\left(1+\alpha_{u}\left(k / k_{s}\right)^{-2 / 3}\right)^{-1 / 2}$, and $\alpha_{u}$ and $\alpha_{\Theta}$ will not be independent.
} 
[14, 16, 17], i.e., the temperature is subjected to a strong shear. This wind is of course caused by buoyancy and somehow a reminiscence of the convection rolls for lower $R a$. Yet it may well be that the temperature in the interior can be considered as a passive scalar. This view is supported by the observation, that the local heat flux in the middle of the cell is tiny 16, 17, 19.

First, we compare our predictions for the scaling exponents in shear flow with the experimentally measured ones and with those of the Bolgiano-Obukhov (BO59) scaling theory [20], which has become the canonical way to account for the RB scaling exponents in the last years [21], but which has also been criticized [22, 23, 3, 24]. As we see from table 1, the experimental scaling exponents, with their limited accuracy, neither favor the shear flow nor the BO59 explanation. Also the numerical simulations by Benzi et al. [19], though excluding a pure K41 scenario, may not be able to distinguish between the BO59 and the shear scenario. The numerical simulations by Kerr and Malevsky [25] (3D) and by Werne [26] (2D) do not favor BO59.

One can also speculate whether RB flow can be considered as helical flow (for a single realization due to spontaneous symmetry breaking) so that (3) can directly be applied. The effective forcing of the velocity field due to the heating will partly be helical so that, besides the energy flux, a helicity flux will be induced at large scales, resulting in a $\propto k^{-7 / 3}$ spectrum as discussed in Section 1.

In the following we will analyze whether the crossover predictions of the two theories allow us to distinguish between them. Therefore we shortly repeat the main ideas of BO59, which was originally suggested for stably stratified flow [20]. The kernel of the BO59 argument is dimensional analysis. Assuming that $\beta g$ and $\epsilon_{\Theta}$ are the relevant parameters, where $\beta$ and $g$ are the volume expansion coefficient and the acceleration due to gravity, one obtains 20, 27, 21, 23]

$$
E_{u}(k) \sim \epsilon_{\Theta}^{2 / 5}(\beta g)^{4 / 5} k^{-11 / 5}, \quad E_{\Theta}(k) \sim \epsilon_{\Theta}^{4 / 5}(\beta g)^{-2 / 5} k^{-7 / 5}
$$

for the velocity and temperature wave vector spectra. If, instead, $\epsilon_{\Theta}$ and the kinetic energy dissipation rate $\epsilon_{u}$ are the relevant parameters, one retrieves Kolmogorov's classical result (11). The crossover from BO59 to K41 is expected for wave vectors $k>k_{B}$, where the Bolgiano scale $k_{B}$ [20, 27] is obtained by equating (1) and (6),

$$
k_{B}=\epsilon_{u}{ }^{-5 / 4} \epsilon_{\Theta}^{3 / 4}(\beta g)^{3 / 2} .
$$

With the exact result [22, 24]

$$
\epsilon_{u}=\kappa^{3} L^{-4} \operatorname{Pr} R a(N u-1), \quad \epsilon_{\Theta}=\kappa \Delta^{2} L^{-2} N u,
$$

the Bolgiano scale $k_{B}$ can be calculated. Here, $N u=H /\left(\kappa \Delta L^{-1}\right)$ is the Nusselt number, $H$ the heat flux $\left(=\left\langle u_{3} \Theta\right\rangle\right.$ in the non diffusive case), $\operatorname{Pr}=\nu / \kappa$ the Prandtl number, $R a=\beta g \Delta L^{3} /(\nu \kappa)$ the Rayleigh number, $L$ the height of the Rayleigh-Benard cell, $\Delta$ the temperature difference between the top and the bottom plate, $\nu$ the viscosity, and $\kappa$ the diffusivity. For large $N u$ we obtain from eq. (可) $k_{B}^{-1}=\operatorname{Pr}^{-1 / 4} R a^{-1 / 4} N u^{1 / 2} L$. The K41 range in the spectra should show up in between the BO59 range and the viscous range, if $k_{B}^{-1}$ is larger than the typical crossover scale to the viscous subrange. For the velocity 
structure function this scale is known [27, 28] to be about $10 \eta$, where $\eta=\nu^{3 / 4} \epsilon_{u}{ }^{-1 / 4}=$ $\operatorname{Pr}^{1 / 2} \mathrm{Ra}^{-1 / 4} N u^{-1 / 4} \mathrm{~L}$ is the Kolmogorov length [27]. For temperature structure functions and high $\operatorname{Pr}$ one could argue that $k_{B}^{-1}$ should be compared with $10 \eta_{\Theta}$, where $\eta_{\Theta}=\operatorname{Pr}^{-1 / 2} \eta$ is the thermal Kolmogorov length [27, 29], which characterizes the crossover between the Batchelor regime (predicted for high $P r$ in a passive scalar intensity spectrum [27, 29]) and the VSR. For very low Prandtl number $\operatorname{Pr}^{-3 / 4} \eta$ should be used [27, 29]. For existing experiments the resulting differences in the $\operatorname{Pr}$ dependence cannot be detected anyhow as $\operatorname{Pr}$ is always roughly 1 , so this difference is only of theoretical interest for the moment. In what follows we will compare $k_{B}^{-1}$ with $10 \eta$ and give the $\operatorname{Pr}$ dependences resulting from a comparison of $k_{B}^{-1}$ with $10 \eta_{\Theta}$ (i.e., the large $\operatorname{Pr}$ case) in brackets.

The condition $k_{B}^{-1}>10 \eta$ for the appearance of the K41 range in between the BO59 and the viscous range requires that

$$
N u>10^{4 / 3} \operatorname{Pr} \approx 22 \operatorname{Pr}
$$

$\left[22 \operatorname{Pr}^{1 / 3}\right.$ when using $\eta_{\Theta}$ instead of $\left.\eta\right]$. I.e., beyond a certain crossover Rayleigh number $R a_{C O}$, defined by $k_{B}^{-1}=10 \eta$, there will be three ranges. For the Helium $(\operatorname{Pr}=0.7)$ convection experiment [15], $N u=0.165 R a^{2 / 7}$, or, embodying the suggested [3] [14, 24] $\mathrm{Pr}$ dependence, $N u=0.157 \mathrm{Pr}^{-1 / 7} \mathrm{Ra}^{2 / 7}$. Thus, if the BO59 scenario were true, pure BO59 scaling should be seen only for $R a<R a_{C O} \approx 3 \cdot 10^{7} \operatorname{Pr}^{4} \approx 10^{7}\left[3 \cdot 10^{7} \operatorname{Pr}^{5 / 2}\right.$ for $\eta_{\Theta}$ instead of $\eta$ ], whereas for $R a>R a_{C O} \approx 10^{7}$ also the K41 range should be visible. Instead, pure $P_{\Theta}(\omega) \propto \omega^{-1.3 \pm 0.1}$ scaling is observed at least up to $R a \approx 10^{11}$ [15. The discrepancy between $R a_{C O}$ and $10^{11}$ can only be resolved by allowing for some prefactors in the dimensional relations like eq. (17) which are far beyond the order of 1 . Note that $R a_{C O}$ is near the experimentally observed transition between soft and hard turbulence [13, 14, 15]. Our results predict that only for larger $\operatorname{Pr}$ the BO59 scaling range might become more extended. Note that in this kind of analysis we neglected the height dependence of $\epsilon$ and $\epsilon_{\Theta}$ and thus of $k_{B}$ by employing eqs. (8)

For comparison, we now try to estimate the crossover between SDR and K41 in RB flow. This amounts to a comparison between the typical shear scale

$$
k_{s}^{-1}=\epsilon_{u}{ }^{1 / 2} / s^{3 / 2}
$$

and $10 \eta$.

First, we estimate the shear $s$ in RB flow. We do so by using the experimental information on the large scale velocity $U=0.16 \kappa L^{-1} R a^{1 / 2}$ [15] and on the mixing layer length $\lambda_{m}$, namely, $s=U / \lambda_{m}$. Originally the mixing layer has been theoretically introduced [14] and later visualized [30] as a region between the thermal boundary layer and the bulk of

\footnotetext{
${ }^{3}$ The Shraiman-Siggia theory [22, 24] assumes $\operatorname{Pr} \gg 1$, but it has proven to work also for smaller $\operatorname{Pr}$. Yet all predictions on $\operatorname{Pr}$ dependences should be taken with care.

${ }^{4}$ The nonuniversal spectral behavior for large $\omega$ and $R a>10^{11}$ 15. could consistently be accounted for by considering the reduced high frequency resolution of the temperature probe because of its finite size [28].

${ }^{5}$ In the numerical simulations of Benzi et al. [19], a height dependence of $k_{B}$ is revealed. These simulations employ periodic boundary conditions. BO59 scaling is found near the boundary (where $k_{B}$ is large), but in contrast to experiment [15] not in the center of the cell.
} 
turbulence. $\lambda_{m} / L \propto R a^{-1 / 7}$ is found [14, 15]. Embodying the predicted $\operatorname{Pr}$ dependence [24], this can be extended to $\lambda_{m} / L \propto \operatorname{Pr}^{4 / 7} R a^{-1 / 7}$. In recent experiments [16, [17] $\lambda_{m}$ was redefined as distance of the maximum of the large scale velocity to the wall. In the employed $L=15.2 \mathrm{~cm}$ RB cell [16, 17], a reduced high frequency resolution of the temperature probe (which is employed to measure $\lambda_{m}$, for the tricky experimental details, see ref. [17]) should be expected around $R a_{a}=\left(100 L^{2} / a^{2}\right)^{4 / 3}$ [28], where $a=200 \mu m$ is the extension of the probe. Indeed, for $R a<R a_{a}$ the measured [17 mixing length $\lambda_{m}$ agrees [31] with the suggested $R a$ scaling law $\lambda_{m} \approx 1.2 \operatorname{LPr}^{4 / 7} R a^{-1 / 7}$. We can thus finally estimate

$$
s=U / \lambda_{m} \approx 0.1 \kappa L^{-2} \operatorname{Pr}^{-4 / 7} \operatorname{Ra}^{9 / 14} .
$$

We now plug (11) and (8) into (10) and obtain $k_{s}^{-1} \approx 8 \operatorname{LPr}^{9 / 7} \operatorname{Ra}^{-9 / 28}$. When comparing $k_{s}^{-1}$ to the crossover $10 \eta$ [or $10 \eta_{\Theta}$ ] to the dissipation dominated range, we get the remarkable result, that the $R a$ scaling of $k_{s}^{-1}$ and $10 \eta$ is the same, namely

$$
k_{s}^{-1} / 10 \eta \approx 0.5 \operatorname{Pr}^{3 / 4}
$$

$\left[0.5 \operatorname{Pr}^{5 / 4}\right.$ for $\eta_{\Theta}$ instead of $\left.\eta\right]$ for all Ra. I.e., for such small $\operatorname{Pr}$ as in the He cell $(\operatorname{Pr}=0.7)$ the shear dominated range is directly followed by the dissipation range with no K41 range in between. For larger $\operatorname{Pr}$ the K41 range might be observable.

Although the shear theory crossover scenario seems to agree better with experiment than the one for BO59 (for small $\operatorname{Pr}=0.7$ ), many questions remain open and our analysis is far from conclusive, as it does not go beyond dimensional analysis. Moreover, the estimation $s=U / \lambda_{m}$ in eq. (11) can be criticized, because the shear might be less in the center of the cell where $P_{\Theta}(\omega)$ is measured. Also, the $z$-dependences of the energy dissipation rate $\epsilon_{u}$ and of the shear $s$ have been neglected. The reason for this section is only to demonstrate that the BO59 scenario is not completely conclusive, either and that the shear scenario might well be able to account for the experimental observations.

\section{Summary}

To summarize, we derive the low frequency $P_{\Theta}(\omega) \propto \omega^{-4 / 3}$ power law for passive scalars in a shear flow [10]. It should be followed by a K41 range (for high degree of turbulence) and of course by a viscous range. This scenario might also account for the measured temperature power spectra in RB flow [15, possibly without the K41 range in between SDR and VSR for low Pr. One of many open questions is the manner in which higherorder moments scale. Benzi et al.'s [19] numerics show strong intermittent behavior, so dimensional analysis would fail here. We hope that this work motivates the analysis of shear effects for both the velocity and the temperature field in numerical simulations and in experiment.

Acknowledgements: We thank R. Almgren, R. Benzi, F. Cattaneo, G. Falkovich, S. Grossmann, L. Kadanoff, A. Müller-Groeling, B. Shraiman, J. Wang, and J. Werne for 
discussions and A. Tilgner and V. Yakhot for supplying us with the results of their work prior to publication. The author also thanks the Aspen Center of Physics for its hospitality. Support by a NATO grant through the Deutsche Akademische Austauschdienst (DAAD), and by DOE is acknowledged.

\section{Table}

\begin{tabular}{|r|r|r|r|r|}
\hline & K41 & BO59 & shear flow & experiment \\
\hline$P_{u}(\omega)$ & $-5 / 3$ & $-11 / 5=-2.2$ & $-7 / 3=-2.33$ & $-2.2 \pm 0.2[32$ \\
$P_{\Theta}(\omega)$ & $-5 / 3$ & $-7 / 5=-1.4$ & $-4 / 3=-1.33$ & $-1.3 \pm 0.1[15]$ \\
\hline
\end{tabular}

Comparison of the scaling exponents predicted by different theories. The last column shows the experimental results. Tong and Shen's [32] refer to velocity structure functions at $R a=10^{9}-10^{10}$. I estimated the experimental error to be at least 0.2 . Note that the very precisely measured $P_{\Theta}(\omega)$ scaling exponent [15] better agrees with the shear flow scenario than with BO59.

\section{Figure}

Figure 1: Temperature power spectra in shear dominated flow, measured behind a wake of a heated cylinder. The data are taken from Sreenivasan's work [10 with kind permission of the author. The spectrum shows a $-4 / 3$ power law. The dashed line shows the shear crossover frequency $s$. In the inset the power spectrum, multiplied by $\omega^{4 / 3}$, is shown. 
* On leave of absence from Fachbereich Physik, Universität Marburg, Renthof 6, D-35032 Marburg.

\section{References}

[1] A. N. Kolmogorov, CR. Acad. Sci. USSR. 30, 299 (1941).

[2] A. M. Obukhov, Izv. Akad. Nauk SSSR, Ser. Geog. Geofiz. 13, 58 (1949); S. Corrsin, J. Appl. Phys. 22, 469 (1951).

[3] S. Grossmann and D. Lohse, Phys. Rev. A 46, 903 (1992).

[4] J. L. Lumley, Phys. Fluids 10, 855 (1967); H. Tennekes and J. L. Lumley, A first course in turbulence (The MIT Press, Cambridge, Massachusetts, 1972).

[5] E. A. Kuznetsov and V. S. L'vov, Physica D 2, 203 (1981).

[6] S. Grossmann, D. Lohse, V. L'vov, and I. Procaccia, Phys. Rev. Lett. 73, 432 (1994).

[7] V. Yakhot, Phys. Rev. E 49, 2887 (1994).

[8] S. G. Saddoughi and S. V. Veeravalli, J. Fluid Mech. 268, 333 (1994).

[9] K. J. Maloy and W. Goldburg, Phys. Fluids A 5, 1439 (1993).

[10] K. Sreenivasan, Proc. R. Soc. London A 434, 165 (1991).

[11] G. I. Taylor, Proc. R. Soc. London A 164, 476 (1938).

[12] D. Lohse and A. Müller-Groeling, "Anisotropy and scaling corrections in turbulence", preprint, Chicago and Toronto 1994.

[13] F. Heslot, B. Castaing, and A. Libchaber, Phys. Rev. A 36, 5870 (1987); M. Sano, X. Z. Wu, and A. Libchaber, Phys. Rev. A 40, 6421 (1989); X. Z. Wu, L. Kadanoff, A. Libchaber, and M. Sano, Phys. Rev. Lett. 64, 2140 (1990).

[14] B. Castaing, G. Gunaratne, F. Heslot, L. Kadanoff, A. Libchaber, S. Thomae, X. Z. Wu, S. Zaleski, and G. Zanetti, J. Fluid Mech. 204, 1 (1989).

[15] I. Procaccia, E. S. C. Ching, P. Constantin, L. P. Kadanoff, A. Libchaber, and X. Z. Wu, Phys. Rev. A 44, 8091 (1991). Besides the PhD thesis by X. Z. Wu, "Along a road to developed turbulence: Free thermal convection in low temperature He gas", University of Chicago, 1991, this reference contains the completest collection of experimental data of the Chicago Rayleigh-Benard experiment.

[16] A. Tilgner, A. Belmonte, and A. Libchaber, Phys. Rev. E 47, 2253 (1993).

[17] A. Belmonte, A. Tilgner, and A. Libchaber, Phys. Rev. Lett. 70, 4067 (1993); Phys. Rev. E 50, 269 (1994). 
[18] F. Chilla, S. Ciliberto, C. Innocenti, and E. Pampaloni, Europhys. Lett. 22, 23 (1993); F. Chilla, S. Ciliberto, and C. Innocenti, Europhys. Lett. 22, 681 (1993).

[19] R. Benzi, R. Tripiccione, F. Massaioli, S. Succi and S. Ciliberto, Europhys. Lett. 25, 341 (1994).

[20] R. Bolgiano, J. Geophys. Res. 64, 2226 (1959); A. M. Obukhov, Sov. Phys. Dokl. 4, 61 (1959).

[21] I. Procaccia and R. Zeitak, Phys. Rev. Lett. 62, 2128 (1989); V. S. L'vov, Phys. Rev. Lett. 67, 687 (1991); V. S. L'vov and G. A. Falkovich, Physica D 57, 85 (1992); V. Yakhot, Phys. Rev. Lett. 69, 769 (1992); S. Grossmann and V. S. L'vov, Phys. Rev. E 47, 4161 (1993).

[22] B. I. Shraiman and E. D. Siggia, Phys. Rev. A 42, 3650 (1990).

[23] S. Grossmann and D. Lohse, Phys. Rev. Lett. 67, 445 (1991).

[24] E. D. Siggia, Annu. Rev. Fluid Mech. 26, 137 (1994).

[25] R. Kerr, "Rayleigh number scaling in Numerical Convection" J. Fluid Mech., submitted (1994); A. V. Malevsky, preprint, University of Minneapolis, 1994.

[26] J. Werne, Phys. Rev. E 48, 1020 (1993) and references therein.

[27] A. S. Monin and A. M. Yaglom, Statistical Fluid Mechanics (The MIT Press, Cambridge, Massachusetts, 1975).

[28] S. Grossmann and D. Lohse, Phys. Lett. A 173, 58 (1993).

[29] G. K. Batchelor, J. Fluid Mech. 5, 113 (1959); S. Grossmann and D. Lohse, Europhys. Lett. 27, 347 (1994).

[30] G. Zocchi, E. Moses, and A. Libchaber, Physica A 166, 387 (1990).

[31] I thank L. P. Kadanoff for this hint (Oct. 1993).

[32] P. Tong and Y. Shen, Phys. Rev. Lett. 69, 2066 (1992). 